\title{
The influence of micromechanical properties and reinforcement architecture on the mechanical response of recycled composites
}

\author{
Soraia Pimenta ${ }^{1, *}$, Silvestre T. Pinho \\ Department of Aeronautics, South Kensington Campus, Imperial College London, SW7 2AZ, United Kingdom
}

\begin{abstract}
Technologies for recycling carbon-fibre composites are now becoming mature, so a key challenge is to establish applications for the recycled products. This paper aims to further our understanding on the mechanical response of recycled composites, so as to guide the optimisation of recycling processes and support their use in non-critical structural components. This work thoroughly characterises three recycled composites and analyses the relations between fibre and interfacial properties, reinforcement architecture, and macroscopic mechanical response. An unequivocal relationship between the architecture of a recycled composite and its fracture toughness is established; this proves that fibre bundles are not necessarily a recycling defect, but can actually toughen the material by at least up to an order of magnitude without significantly reducing stiffness or strength.
\end{abstract}

Keywords: A. Recycling, A. Discontinuous reinforcement, B. Fracture toughness, D. Mechanical testing

\section{Introduction}

The exponential growth in Carbon-Fibre Reinforced-Polymer (CFRP) use has promoted the development of recycling technologies for the waste generated $[1,2]$. This paper analyses the mechanical response of three state-of-the-art recycled composites, focusing on the effect of fibre properties and architecture on fracture toughness.

Recycled ( $\mathrm{r}-$ ) CFRP typically comprise carbon-fibres reclaimed through a thermo-chemical process (most commonly pyrolysis) embedded in a polymeric (e.g. epoxy) matrix; the most common architecture is characterised by a discontinuous and randomly-oriented reinforcement [1]. RCFRPs have been usually characterised at the macro-scale in terms of stiffness and strength [3-8]. In a previous study [9], the authors provided new insight by showing

\footnotetext{
${ }^{*}$ Corresponding author.

Email address: soraia.pimenta@imperial.ac.uk (Soraia Pimenta)

${ }^{1}$ Present address: Department of Mechanical Engineering, South Kensington Campus, Imperial College London, SW7 2AZ, United Kingdom

Pimenta S, Pinho ST (2014). The influence of micromechanical properties and reinforcement architecture on the mechanical response of recycled composites. Composites Part A - Applied Science and Manufacturing 56, 213-225. DOI:10.1016/j.compositesa.2013.10.013
} 
that fibre bundles — held together by residual matrix not completely removed during fibre reclamation - locally toughened the material. However, this effect was limited, as very few bundles survived the fibre disentanglement stage purposely included in the manufacturing process [5].

The toughening effect of bundles in fibre reinforced polymers has been reported in the literature for virgin materials [10-14]. The mechanism is typically justified by an increase on the dissipation of pull-out energy as the scale of reinforcement units grows, assuming constant volume fraction and aspect ratio of the reinforcement. Nevertheless, large fibre bundles are frequently associated with composites with lower stiffness and strength; moreover, bundling is difficult to induce and control in virgin systems [15].

Consequently, the concept of enhancing the toughness of CFRPs with fibre bundles has been mostly explored by researchers only. However, the recent development of several composites with meso-scale discontinuous reinforcement [16-18] has proved that these materials offer not only improved manufacturability, but also compelling properties and damage tolerance.

Fibre bundles are naturally induced in rCFRPs by the fibre reclamation process; pyrolysis — currently the most used method $[1,19-22]$ — is particularly prone to leaving small amounts of residual matrix on the fibre surfaces (unless very aggressive cycles are used). Most re-impregnation techniques involve depositing recycled fibres from a liquid medium onto preforms [3, 23], applicable to bundles as well; filamentising the material, on the other hand, requires extra processing [5].

Different recycling routes can create different meso-scale architectures in rCFRPs, but they are also likely to affect the micromechanical properties of the fibres and fibre-matrix interface $[22,24,25]$. In order to understand how both micro and meso levels affect the macroscopic mechanical properties, it is thus necessary to fully characterise the materials at all three existing scales.

This paper aims at analysing experimentally the relation between (i) micromechanical properties (including fibre morphology, fibre strength and matrix interface), (ii) reinforcement architecture (which are quantitatively characterised), and (iii) mechanical properties (with emphasis on the fracture toughness) of rCFRPs. Three different materials (obtained through distinct recycling routes as described in Section 2) are investigated. The experimental procedures are detailed in Section 3, and results are presented in Section 4. Section 5 discusses the most important findings, and Section 6 sums up the main conclusions.

\section{Materials}

The recycling and re-manufacturing routes for the three rCFRPs analysed are described

in Table 1. All materials feature discontinuous fibres reclaimed through pyrolysis either at Recycled Carbon Fibre Ltd. (now ELG Carbon Fibre Ltd. (ELG-RCF) [19]) or Materials In- 
Table 1: Recycling route of materials studied.

\begin{tabular}{l|ccc|ccr}
\hline & \multicolumn{3}{|c|}{ Fibre reclamation } & \multicolumn{3}{c}{ Re-manufacturing } \\
rCF/ rCFRP & $\begin{array}{c}\text { Waste } \\
\text { source }^{(\star)}\end{array}$ & $\begin{array}{c}\text { Fibre } \\
\text { type }\end{array}$ & $\begin{array}{c}\text { Pyrolysis } \\
\text { process }\end{array}$ & $\begin{array}{c}\text { Preforming } \\
\text { process }^{(\dagger)}\end{array}$ & $\begin{array}{c}\text { Resin } \\
\text { impregnation }\end{array}$ & $\begin{array}{c}\text { Epoxy } \\
\text { resin }\end{array}$ \\
\hline T300-rRCF [9] & uMW & T300 [27] & ELG-RCF & TFP & U.Nottingham & MTM57 [28] \\
T300-rMIT & uMW & T300 [27] & MIT-RCF & MIT-RCF & Boeing & RTM 6 [29] \\
T800-rMIT & EoL & T800 [30] & MIT-RCF & MIT-RCF & Boeing & RTM 6 [29] \\
\hline
\end{tabular}

${ }^{(*)}$ uMW: uncured Manufacturing Waste; EoL: End-of-Life component.

(†) TFP (Technical Fibre Products) used their papermaking technique [23]; MIT-RCF used their 3-D engineered preform process [3].

${ }^{(\dagger)}$ U.Nottingham used compression moulding [5]; Boeing used Resin Transfer Moulding (RTM) [7].

novation Technologies - Reengineered Carbon Fiber (MIT-RCF [20]); while both companies have now industrial-scale processes, the fibres used in this work were reclaimed in earlier smaller-scale implementations [3, 26]. All details of the recycling processes are proprietary.

Material T300-rRCF has been previously analysed by the authors [9]; it is included here for characterisation at the micromechanical level and comparison purposes. Materials T300-rMIT and T800-rMIT were reclaimed and re-manufactured through the same route, while materials T300-rRCF and T300-rMIT share the same waste source. In all cases, manufacturing included preforming and subsequent resin moulding with epoxy matrices and target fibre content at $V^{\mathrm{f}}=30 \%$. The three materials tested (Table 1) combine two distinct waste sources, two distinct recycling processes, and two distinct re-manufacturing processes (with two distinct epoxy matrices). Consequently, the architecture of the reinforcement differed significantly in the three materials, ranging from very fine (with short dispersed fibres) to rather coarse (with large fibre bundles).

Standard mechanical properties of the three recyclates are shown in Figure 1. For material T300-rRCF, both in-plane principal directions are shown [9]. The properties of materials T300-rMIT and T800-rMIT (nominally isotropic in-plane) were measured by the manufacturer [31].

\section{Experimental analysis}

\subsection{Single-fibre analysis}

\subsubsection{Fibre inspection}

The morphologies of all fibre types (virgin and recycled) were investigated through Scanning Electron Microscopy (SEM) of tows (taken from the centre of the reclaimed mats). Fibre diameters $\left(\phi^{\mathrm{f}}\right)$ were measured at 4,000× magnification in 25 filaments.

\subsubsection{Single fibre tensile tests}

The tensile strength of all fibre types were determined through Single-Fibre Tensile Tests (SFTTs) $[22,32]$ performed at two gauge lengths, following the specifications shown in Table 2. 


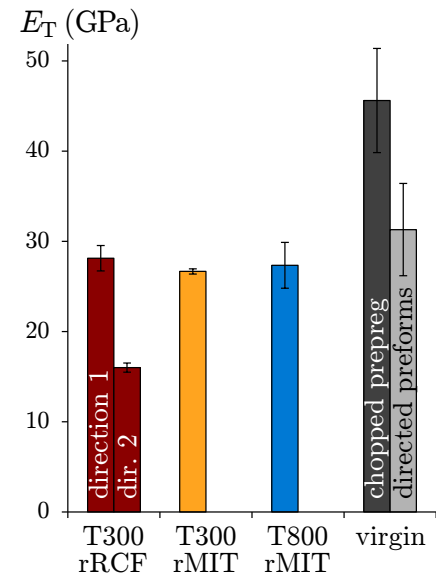

(a) Young's modulus.

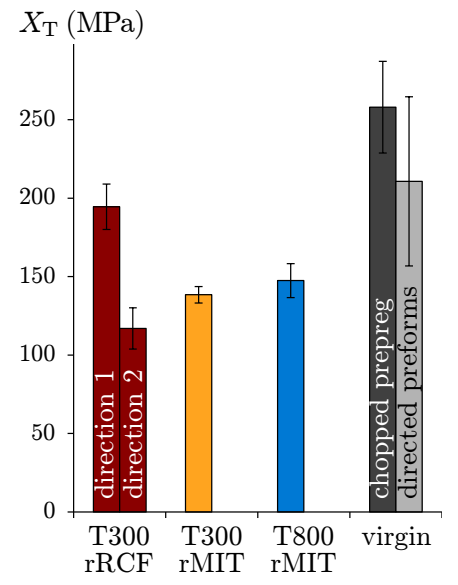

(b) Tensile strength.

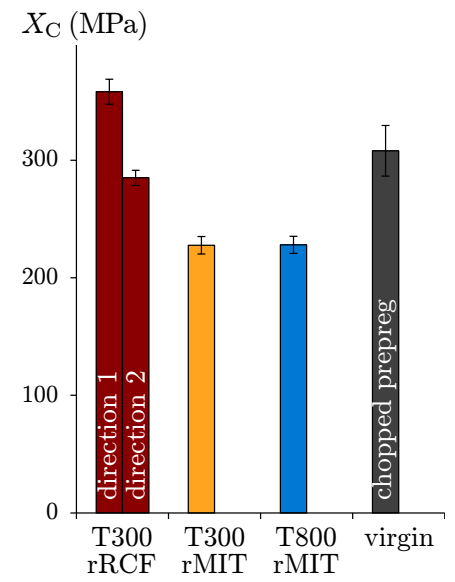

(c) Compressive strength.

Figure 1: Standard mechanical properties of the three rCFRPs under investigation and similar virgin materials from the literature. Material T300-rRCF has been previously characterised [9]; directions 1 and 2 are respectively aligned with and normal to the preferential fibre direction. Materials T300-rMIT and T800-rMIT were characterised by Pete E George at Boeing Research and Technology. Virgin materials correspond to stateof-the-art discontinuous carbon-epoxy systems, manufactured with chopped prepreg $\left(V^{\mathrm{f}} \approx 60 \%[17]\right)$ and Directed Carbon Fibre Preforming $\left(V^{\mathrm{f}} \approx 40 \%[18]\right)$; values shown here were averaged over different specimen sizes.

Table 2: Specifications for single-fibre tensile tests.

\begin{tabular}{ccccc}
\hline $\begin{array}{c}\text { Load } \\
\text { cell }\end{array}$ & $\begin{array}{c}\text { Type } \\
\text { of glue }\end{array}$ & $\begin{array}{c}\text { Fibre gauge } \\
\text { length }(\mathrm{mm})\end{array}$ & $\begin{array}{c}\text { Displacement } \\
\text { rate }(\mathrm{mm} / \mathrm{min})\end{array}$ & $\begin{array}{c}\text { Number of tests } \\
\text { (per fibre type) }\end{array}$ \\
\hline \multirow{2}{*}{$10 \mathrm{~N}$} & 3M Scotch-Weld & 10 & 0.1 & $\geq 20$ \\
& 9323 B/A epoxy & 20 & 0.2 & $\geq 20$ \\
\hline
\end{tabular}

Individual realisations of fibre strength $\left(\sigma^{\mathrm{f}}\right)$ were calculated using the average diameter of the corresponding fibre type.

The shape $(m)$ and scale $\left(\sigma_{0}^{f}\right)$ Weibull parameters for the strength distribution of each fibre type and at each gauge length were estimated through the maximum likelihood method [22]. In addition, the size-effect Weibull modulus was calculated from the scale parameters $\sigma_{0,1}^{\mathrm{f}}$ and $\sigma_{0,2}^{\mathrm{f}}$, estimated at the respective gauge lengths $l_{1}$ and $l_{2}$ :

$$
\frac{\sigma_{0,1}^{\mathrm{f}}}{\sigma_{0,2}^{\mathrm{f}}}=\left(\frac{l_{2}}{l_{1}}\right)^{1 / m} \Longrightarrow m=\frac{\ln \left(l_{2} / l_{1}\right)}{\ln \left(\sigma_{0,1}^{\mathrm{f}} / \sigma_{0,2}^{\mathrm{f}}\right)}
$$

This definition has the advantage of segregating the spurious effect of test variability within a gauge length, although it is susceptible to end-effects [33]. 
Table 3: Specifications for single-fibre pull-out tests.

\begin{tabular}{cccccc}
\hline $\begin{array}{c}\text { Load } \\
\text { cell }\end{array}$ & $\begin{array}{c}\text { Embedding matrix } \\
\text { diameter }\end{array}$ & $\begin{array}{c}\text { Displacement } \\
\text { rate }\end{array}$ & $\begin{array}{c}\text { Fibre } \\
\text { type }\end{array}$ & $\begin{array}{c}\text { Embedded fibre } \\
\text { length }(\mu \mathrm{m})\end{array}$ & $\begin{array}{c}\text { Valid } \\
\text { tests }^{(\star)}\end{array}$ \\
\hline & & & T300-v & $22-86$ & 10 \\
$50 \mathrm{~N}$ & \multirow{2}{*}{$3.5 \mathrm{~mm}$} & \multirow{2}{*}{$0.06 \mathrm{~mm} / \mathrm{min}$} & T300-rRCF & $16-73$ & 10 \\
& & & T300-rMIT & $44-108$ & 2 \\
& & & T800-rMIT & 76 & 1 \\
\hline
\end{tabular}

${ }^{(\star)}$ Within a total of 20 SFPO tests performed for each fibre type.

\subsubsection{Single-fibre pull-out tests}

The properties of the fibre-matrix interface were estimated through Single-Fibre Pull-Out (SFPO) tests, using a setup described elsewhere [34] and the specification defined in Table 3. For each type of composite, individual fibres were partially embedded in a matrix block and cured according to the resin manufacturer's instructions; subsequently, the free end of each embedded fibre was pulled-out under displacement control, and the load-displacement curve, $P(u)$, was recorded. The apparent interfacial shear strength $\left(S_{\mathrm{IF}}\right)$ and fibre-matrix frictional stress $\left(\tau_{\mu}^{0}\right)$ were calculated using the average diameter of the corresponding fibre type as [35]:

$$
S_{\mathrm{IF}}=\frac{P^{\max }}{\pi \cdot \phi^{\mathrm{f}} \cdot l_{\mathrm{po}}} \quad \text { and } \quad \tau_{\mu}^{0}=\frac{\mathrm{d} P}{\mathrm{~d} u}\left(l_{\mathrm{po}}\right) \cdot \frac{1}{\pi \cdot \phi^{\mathrm{f}}}, \quad \text { with } \quad l_{\mathrm{po}}=\{u: P(u)=0\} .
$$

The RTM6 matrix exhibited significant shrinkage during cure, which made it difficult to prepare T300-rMIT and T800-rMIT samples with suitable embedded lengths. The free surface of the matrix moved considerable while the fibre was still attached to the embedding rig, thus releasing the embedded fibre end. For that reason, longer embedding lengths had to be used, meaning that very few samples of the T300-rMIT and T800-rMIT materials actually pulled-out successfully (respectively 2 and 1 successful tests out of 20 samples).

\subsection{Composite analysis}

\subsubsection{Characterisation of microstructure}

The volume content of fibres $\left(V^{\mathrm{f}}\right)$, resin $\left(V^{\mathrm{m}}\right)$ and voids $\left(V^{\mathrm{v}}\right)$ in each material was measured in 8 randomly selected optical micrographs from two orthogonal through-the-thickness sections; each micrograph comprised an area of $2 \mathrm{~mm}^{2}$ [9].

The architecture of materials T300-rMIT and T800-rMIT was characterised by measuring the bundle length and width distributions $\left(F_{L}(l)\right.$ and $F_{W}(w)$ respectively) in the corresponding dry preforms manufactured by MIT-RCF. This required (i) cutting a square layer (length $\ell$ ) from the preform, (ii) picking and measuring $N_{w}$ individual bundles using image analysis, and (iii) calculating the theoretical median ranks of each realisation $[22,36]$. For bundle length distributions, only those $\left(N_{l}\right)$ bundles not crossing the edges of the $\ell \times \ell$ sample were 
Table 4: Specifications for architecture characterisation.

\begin{tabular}{|c|c|c|c|c|}
\hline Material & $\begin{array}{l}\text { Layer sample size } \\
\quad \ell \times \ell\left(\mathrm{mm}^{2}\right)\end{array}$ & $N_{w}$ & $N_{l}$ & $\begin{array}{c}\text { Weight fraction of } \\
\text { bundles actually } \\
\text { measured }\end{array}$ \\
\hline T300-rMIT & \multirow{2}{*}{$100 \times 100$} & 1596 & 1038 & $80 \%$ \\
\hline T800-rMIT & & 2407 & 1809 & $73 \%$ \\
\hline
\end{tabular}

included; as this creates a bias towards shorter bundles, the rank of each observation $i$ was corrected as suggested by Fu et al. [37]:

$$
i^{\prime}=\sum_{j=1}^{i}\left(\frac{8 \cdot \ell \cdot l_{i}}{\pi \cdot \ell^{2}-4 \cdot \ell \cdot l_{i}+\left(l_{i}\right)^{2}}+1\right) .
$$

This process considered all bundles in the sample approximately wider than $0.1 \mathrm{~mm}$; thinner bundles represented only a small weight fraction of the dry preforms and were were not evaluated. The specifications for the measurements are shown in Table 4.

The architecture of material T300-rRCF was assumed to be the same as that measured by Wong et al. [5] for a very similar material (only differing from T300-rRCF in the epoxy matrix used for re-impregnation). Only the dispersed phase was considered, as a fibre disentanglement step during manufacture had filamentised most bundles [5].

\subsubsection{Compact tension tests}

The tensile fracture toughness $\mathcal{G}$ of materials T300-rMIT and T800-rMIT was measured through Compact Tension (CT) tests [38], following a similar procedure as used previously for material T300-rRCF [9]. The pre-crack in all geometries (Figure 2) was cut with a wire saw (approximately $0.6 \mathrm{~mm}$ wide) and sharpened with a surgical blade; specimens were equipped with an extensometer mounted at two lateral slots, and tested under displacement control (at $0.5 \mathrm{~mm} / \mathrm{min}$ ) through loading pins.

The load $P$, cross-head displacement $d_{\text {xhead }}$ and extensometer opening $d_{\text {ext }}$ were recorded during the test. The data reduction was based on compliance calibration through linearelastic Finite Elements (FE) models [38, 39]:

1. For each specimen geometry, material and crack length $a \in\left[a_{0}, a_{0}+\Delta a^{\max }\right]$, the relations $a_{\mathrm{FE}}\left(C^{\text {extens }}\right), C_{\mathrm{FE}}^{\text {pins }}(a)$ and $J_{\mathrm{FE}}^{\text {unit }}(a)$ (where $C$ is the unit-thickness compliance and $J_{\mathrm{FE}}^{\text {unit }}$ is the J-integral for unit load $P$ and unit specimen thickness $t$ ) were extracted through $\mathrm{FE}$;

2. The initial linear-elastic domain in each test (represented by the subscript 0 ) was used to calibrate the effective initial crack length as $a_{0}=a_{\mathrm{FE}}\left(C_{0}^{\text {extens }}\right)$, and the compliance of the setup as $C^{\text {setup }}=C_{0}^{\text {xhead }}-C_{\mathrm{FE}}^{\text {pins }}\left(a_{0}\right)$. It was experimentally verified that 

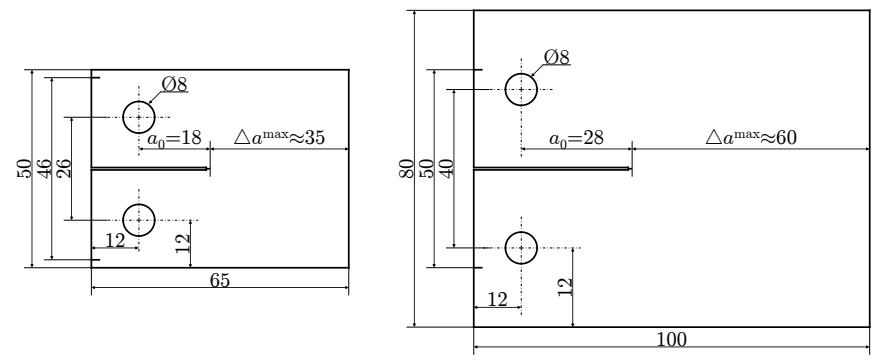

(a) Type A.

(b) Type B (T800-rMIT only).

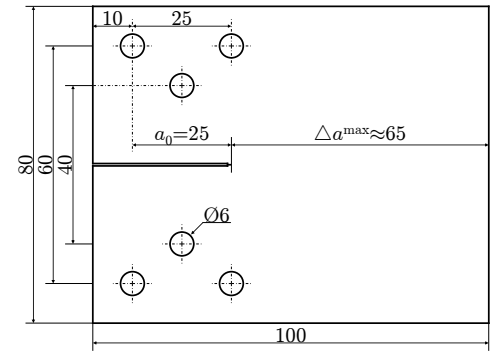

(c) Type C (T800-rMIT only).

Figure 2: Geometry of CT specimens for materials T300-rMIT and T800-rMIT (the toughness of material T300-rRCF had been previously measured [9]).

$$
C^{\text {setup }} / C_{0}^{\text {xhead }} \approx 15 \%
$$

3. For the entire duration of each test, the $\mathrm{R}$-curves were calculated through

$$
a=a_{\mathrm{FE}}\left(C^{\text {pins }}\right) \text { and } \mathcal{G}=J_{\mathrm{FE}}^{\text {unit }}\left(C^{\text {pins }}\right) \cdot P^{2} / t^{2} \text {, with } C^{\text {pins }}=C^{\text {xhead }}-C^{\text {setup }} .
$$

Tests were initially conducted with specimens as shown in Figure 2a (hereafter designated type A). However, material T800-rMIT could not fully develop a process zone in type-A specimens, and the crack followed a reasonably straight path in one test only. A larger specimen (type B, Figure $2 \mathrm{~b}$ ) was attempted, but proved unreliable due to frequent out-ofplane buckling. Finally, a multi-loading-point setup (originally proposed for mixed-mode translaminar toughness [40], but here applied to pure mode-I) was used with satisfactory results; in this case, $a_{0}$ and $C^{\text {setup }}$ were calibrated by tracking directly the displacement of the loading pins with an image recording system (instead of the extensometer).

The tensile toughening mechanisms were investigated in post-mortem fracture surfaces of valid $\mathrm{CT}$ tests.

\section{Results}

\subsection{Single-fibre analysis}

\subsubsection{Fibre morphology}

Diameter measurements of all fibre types analysed are presented in Figure 3. There is no statistically significant difference between the diameters of virgin and recycled T300 fibres (contrarily to the results previously obtained for T300-rRCF [5, 9]). However, the T800-rMIT fibres are significantly larger than the virgin precursors ( $p$-value of $0.0002 \%$ ), suggesting the presence of a residual matrix layer on their surface.

Figure 4 presents SEM images of T300 fibres. Virgin filaments are clean and smooth, apart from the striations characteristic of this fibre type. The surface of recycled fibres presented a low amount of residual matrix, but were otherwise clean and showed no evidence of fibre damage. 


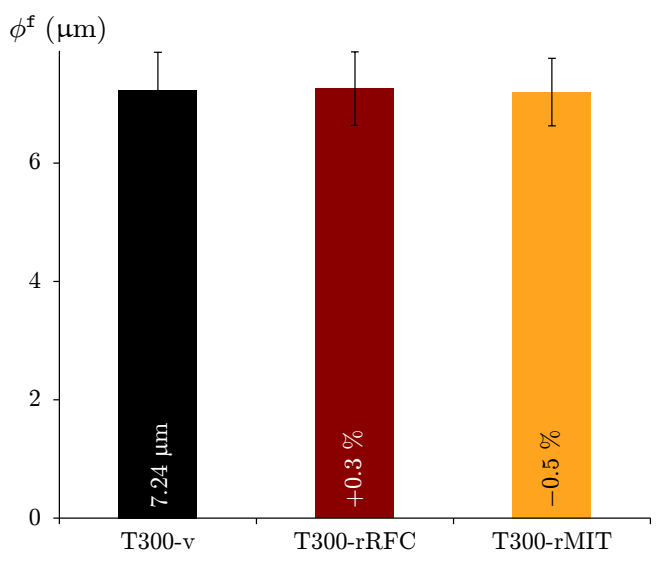

(a) T300 fibre type.

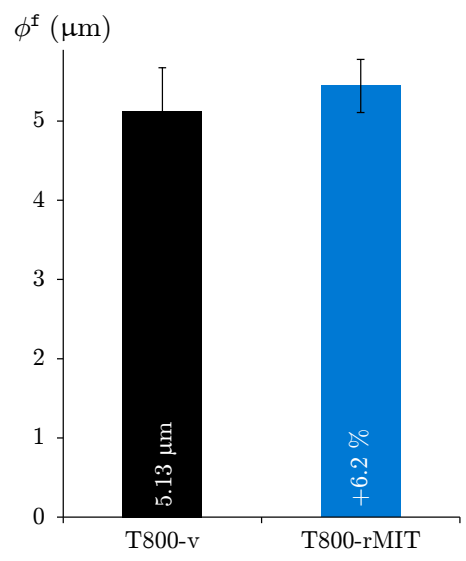

(b) T800 fibre type.

Figure 3: Fibre diameters of virgin and recycled fibres. Average percent reduction (relatively to the virgin fibre) are indicated; error bars represent one standard deviation.

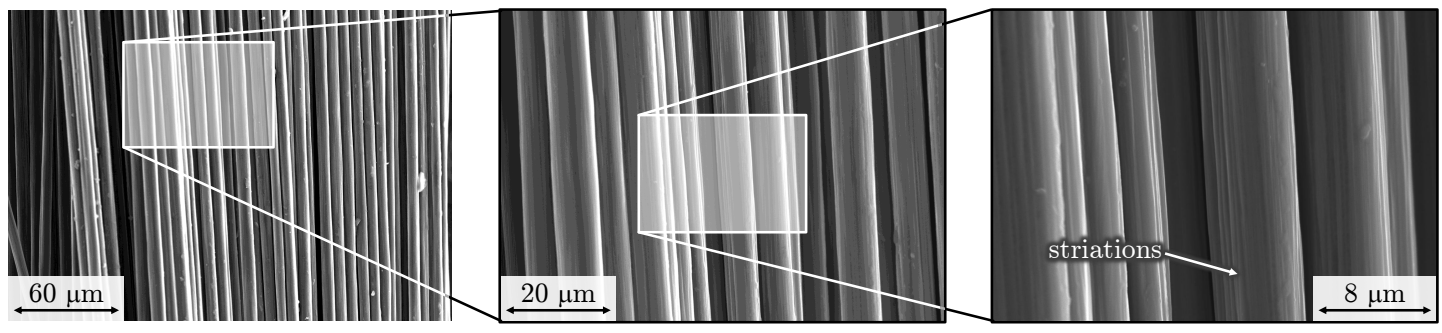

(a) T300-v fibres.

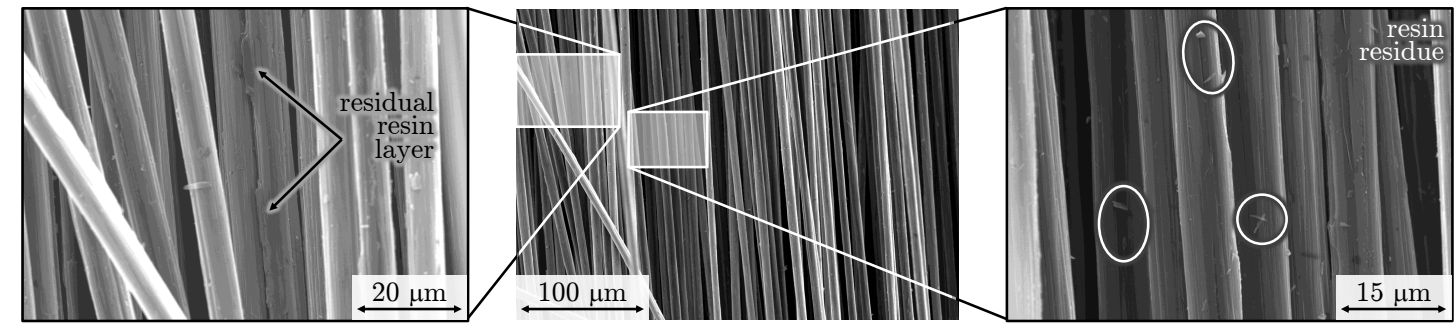

(b) T300-rRCF fibres.

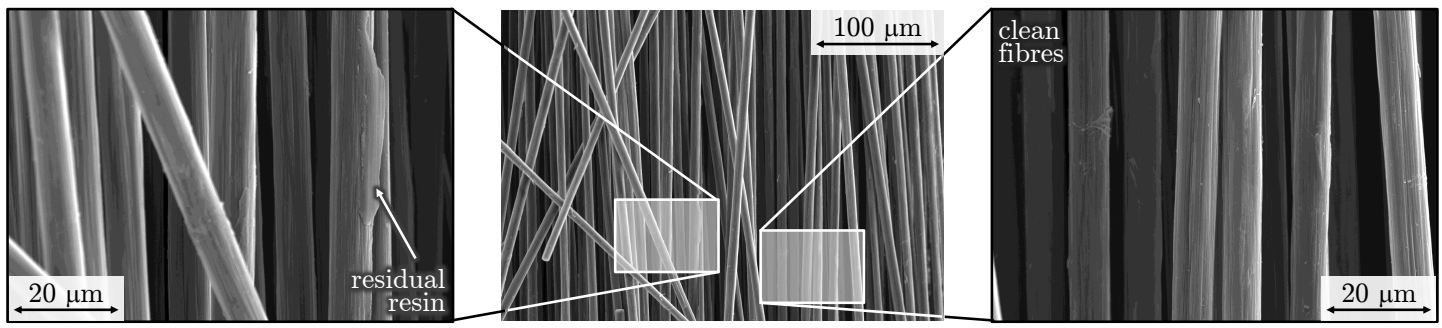

(c) T300-rMIT fibres.

Figure 4: Scanning electron micrographs of virgin and recycled T300 fibres. 


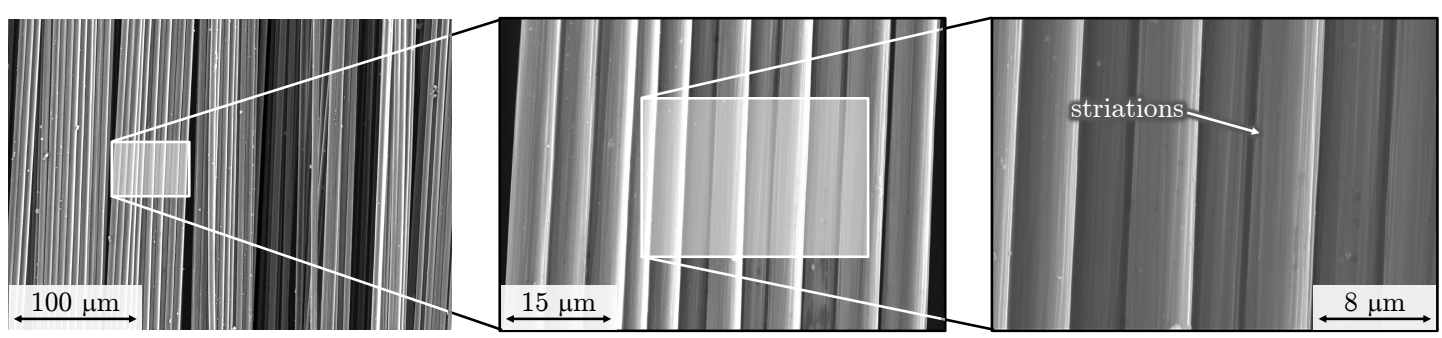

(a) T800-v fibres.
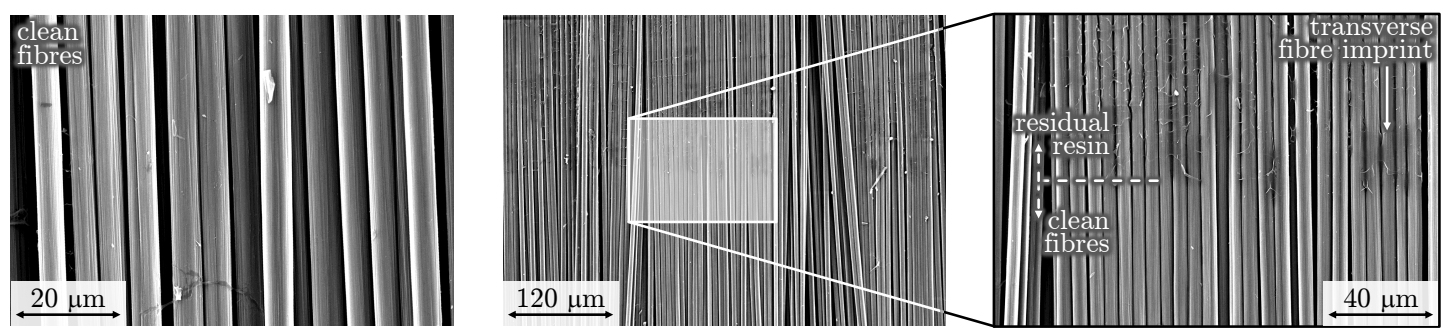

(b) T800-rMIT fibres.

Figure 5: Scanning electron micrographs of virgin and recycled T800 fibres.

The morphology of virgin and recycled T800 fibres is shown in Figure 5. The alternate presence of clean fibres and residual matrix indicates that the waste source of the T800-rMIT recyclate contained woven composites, in which transverse tows protected alternating regions of the weave pattern from the recycling process.

\subsubsection{Single-fibre tensile strength}

Average fibre strengths and corresponding standard deviations are shown in Figure 6. The strength of T300 fibres was not significantly affected by the recycling process. On the contrary, the average strength of T800-rMIT was statistically lower ( $p$-value of $0.1 \%$ ) than that of the virgin type; the difference is nevertheless small, and is actually magnified by the larger diameter of the former (although it still verifies for the (non-normalised) failure load).

Figures 7 and 8 present experimental and fitted single-fibre strength distributions in Weibull plots. The quality of the fitting is good for all fibre types and at both $10 \mathrm{~mm}$ and $20 \mathrm{~mm}$ gauge lengths.

However, Table 5 shows that the Weibull moduli calculated from distributions at each gauge length are not necessarily identical to each other; moreover, they tend to be significantly different from the modulus calculated assuming a Weibull-type size effect (Equation 1). Strength variability in T300 fibres increased only slightly after recycling, while it was actually reduced for the T800 fibre type. In addition, and although size effects are evident in all fibre types, strength retention after recycling was higher for the longer gauge length (Figure 6 and Table 5). 


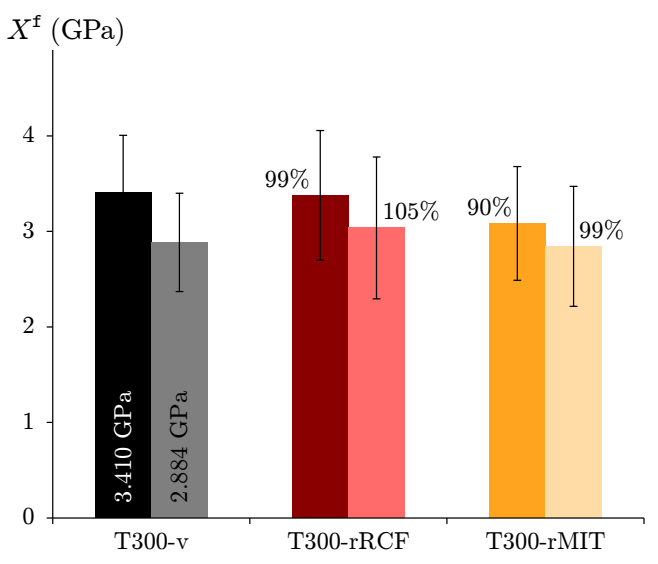

(a) T300 fibre types.

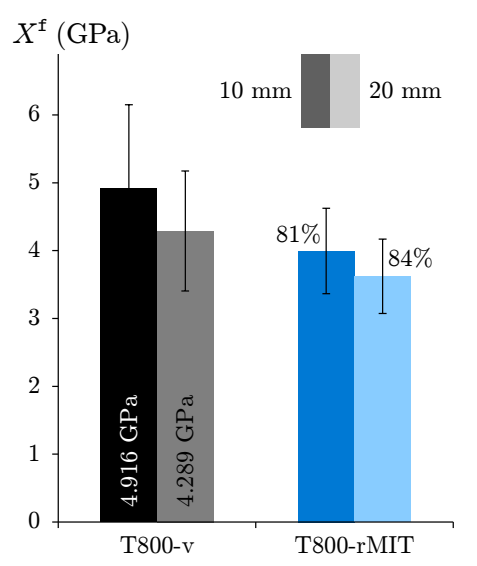

(b) T800 fibre types.

Figure 6: Strength statistics for virgin and recycled fibres. Average strength retentions (relatively to the virgin fibre) are indicated; error bars represent one standard deviation. For each fibre type, the left (darker) and right (lighter) columns correspond to gauge lengths of $10 \mathrm{~mm}$ and $20 \mathrm{~mm}$ respectively.

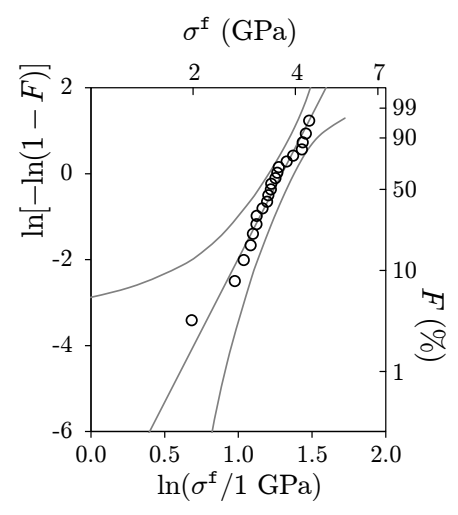

(a) T300-v, $l=10 \mathrm{~mm}$.

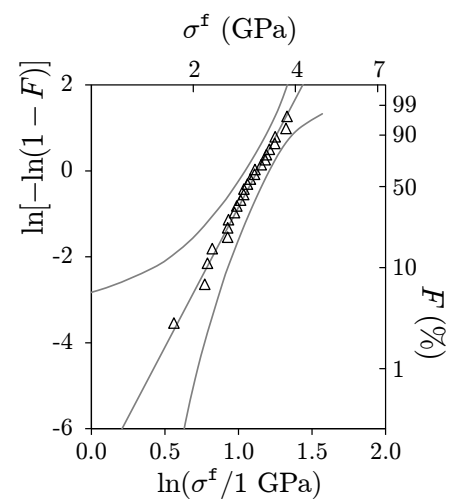

(d) T300-v, $l=20 \mathrm{~mm}$.

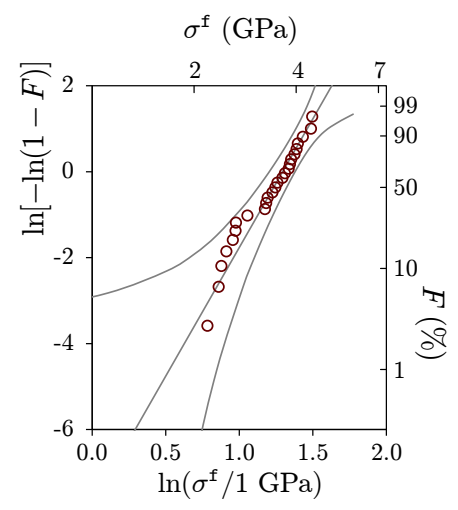

(b) T300-rRCF, $l=10 \mathrm{~mm}$.

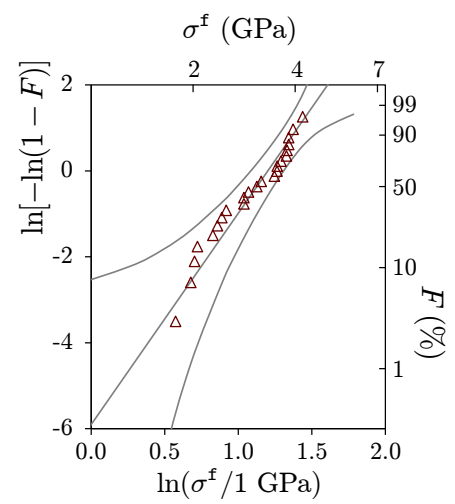

(e) T300-rRCF, $l=20 \mathrm{~mm}$.

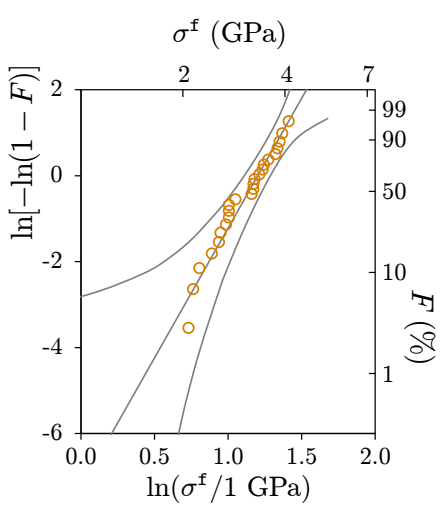

(c) T300-rMIT, $l=10 \mathrm{~mm}$.

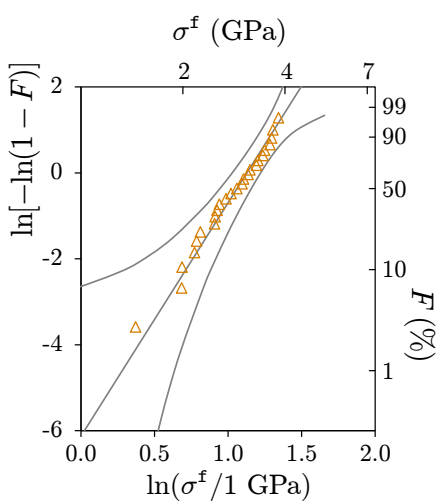

(f) T300-rMIT, $l=20 \mathrm{~mm}$.

Figure 7: Strength distributions of virgin and recycled T300 fibres. 


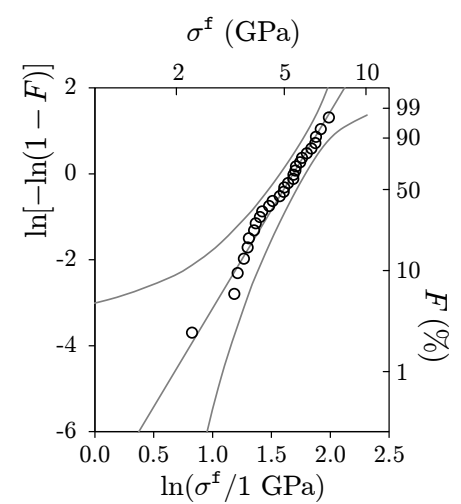

(a) $\mathrm{T} 800-\mathrm{v}, l=10 \mathrm{~mm}$.

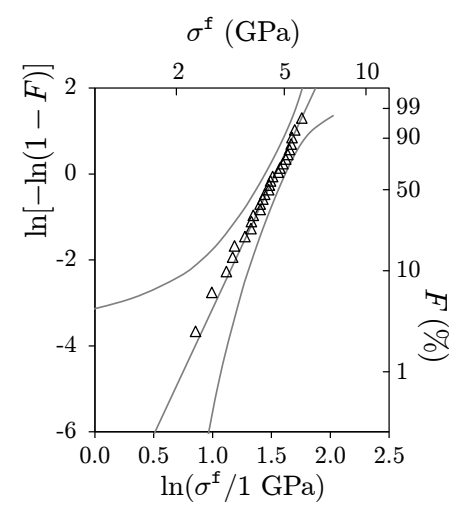

(c) $\mathrm{T} 800-\mathrm{v}, l=20 \mathrm{~mm}$.

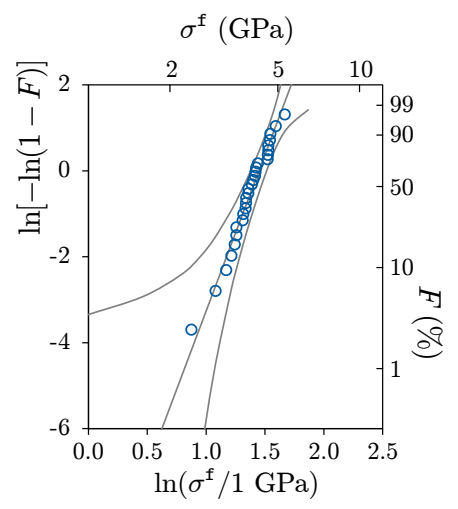

(b) T800-rMIT, $l=10 \mathrm{~mm}$.

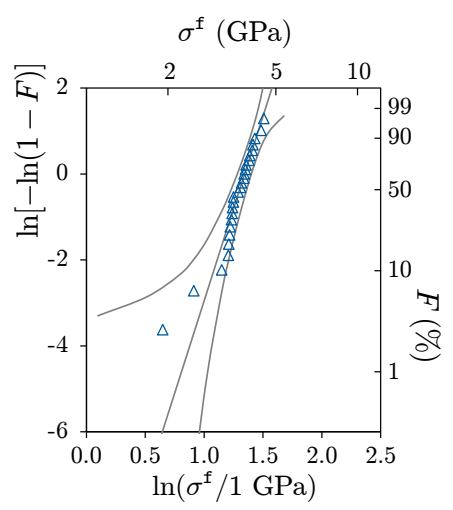

(d) T800-rMIT, $l=20 \mathrm{~mm}$.

Figure 8: Strength distributions of virgin and recycled T800 fibres.

Table 5: Weibull fitting to SFTT strengths.

\begin{tabular}{lccccc}
\hline \multirow{2}{*}{ Fibre type } & \multicolumn{2}{c}{$l=10 \mathrm{~mm}{ }^{(\star)}$} & \multicolumn{2}{c}{$l=20 \mathrm{~mm}{ }^{(\star)}$} & Size effect ${ }^{(\dagger)}$ \\
\hline T300-v & $m(-)$ & $\sigma_{0}^{\mathrm{f}}(\mathrm{GPa})$ & $m(-)$ & $\sigma_{0}^{\mathrm{f}}(\mathrm{GPa})$ & $m(-)$ \\
T300-rRCF & 6.69 & 3.651 & 6.52 & 3.094 & 4.19 \\
T300-rMIT & 5.99 & 3.649 & 4.92 & 3.321 & 7.38 \\
\hline T800-v & 6.04 & 3.326 & 5.43 & 3.089 & 9.38 \\
T800-rMIT & 4.58 & 5.387 & 5.87 & 4.637 & 4.63 \\
\hline
\end{tabular}

${ }^{(\star)}$ Maximum likelihood fitting for individual distributions.

${ }^{(\dagger)}$ Size-effect Weibull modulus (predicted by Equation 1).

\subsubsection{Fibre-matrix interfacial properties}

The interfacial properties measured through SFPO tests are presented in Figure 9. Further analysis revealed a characteristically linear load-displacement curve during the friction stage, as well as very little correlation between $S_{\mathrm{IF}}$ and $l_{\mathrm{po}}$; this supports the use of an average interfacial shear strength and of constant frictional stresses as assumed in Equation 2. 


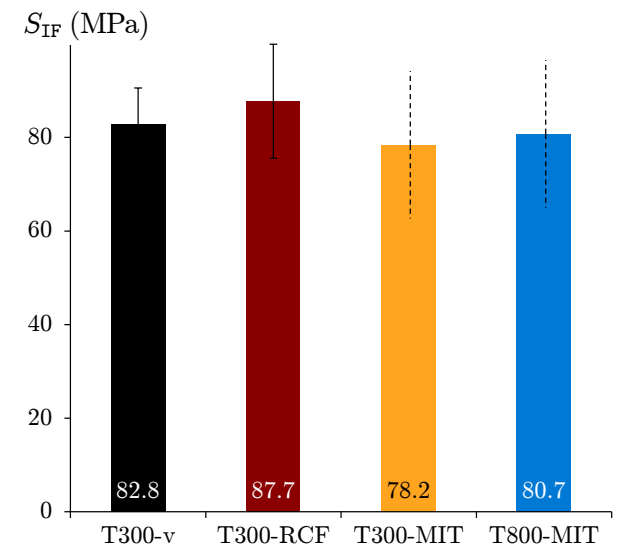

(a) Apparent interfacial shear strength.

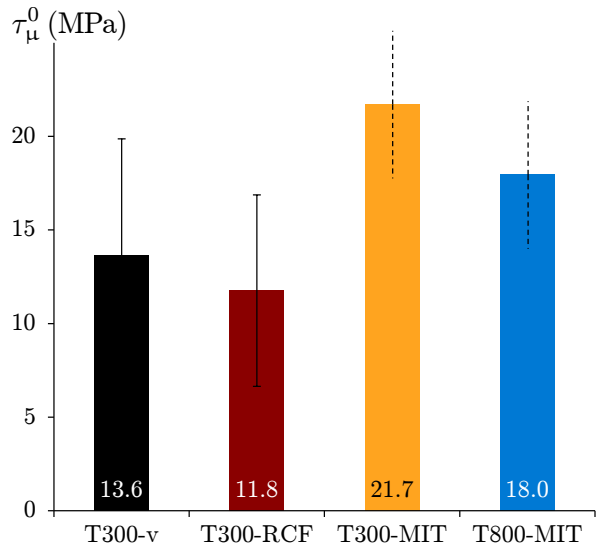

(b) Pull-out frictional stress.

Figure 9: Fibre-matrix interfacial properties obtained from SFPO tests. Error bars represent standard deviations (not available for T300-rMIT and T800-rMIT composites).

Figure 9a suggests that the interfacial adhesion in the T300-rRCF material is at least as good as when virgin fibres are used. The frictional stresses measured in both materials have considerable scatter, but are statistically similar and consistent with literature values [41, 42].

Due to the low number of successful tests (see Section 3.1.3), results for T300-rMIT and T800-rMIT systems in Figure 9 are indicative only; nevertheless, they suggest a good interfacial adhesion in both materials. The higher interfacial frictional stresses measured could be caused by higher residual stresses, a rougher fibre-matrix surface, or simply by statistical variance.

\subsection{Analysis of the composite}

\subsubsection{Constituents and reinforcement architecture}

Figure 10 shows the dry preforms of materials T300-rMIT and T800-rMIT, evidencing bundles on a mat of dispersed fibres; the multiscale character of the reinforcement is obvious in both cases. Material T300-rMIT clearly has a finer architecture; it is also evident that most bundles in material T800-rMIT originate from non-split tows of a woven fabric.

Through-the-thickness optical micrographs of the three materials further highlight the different architectures. Material T300-rRCF (Figure 11a) has most fibres finely dispersed within the matrix and only a few bundles. Material T300-rMIT (Figure 11b) presents a continuous range of bundle sizes. Material T800-rMIT (Figure 11c) shows very large and compact bundles, and fewer dispersed fibres.

Figure 12 suggests that fibre content is slightly higher in the materials manufactured at Boeing than at the University of Nottingham. Material T800-rMIT has the highest void content, likely due to the presence of residual resin (which has been shown to obstruct resin flow and impregnation [22]). 


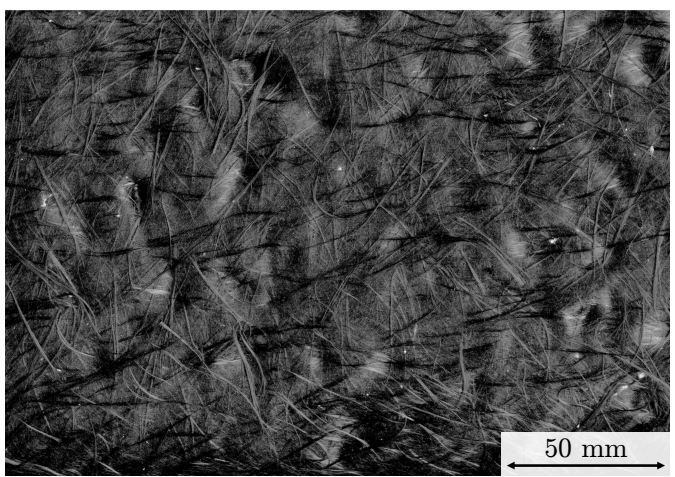

(a) T300-rMIT.

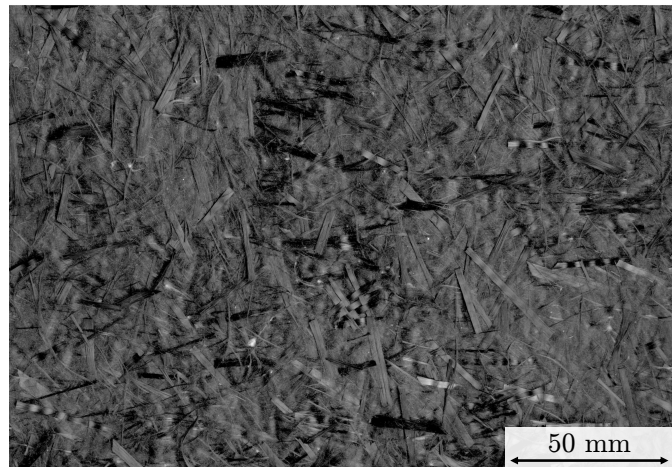

(b) T800-rMIT.

Figure 10: Dry preforms of the T300-rMIT and T800-rMIT materials

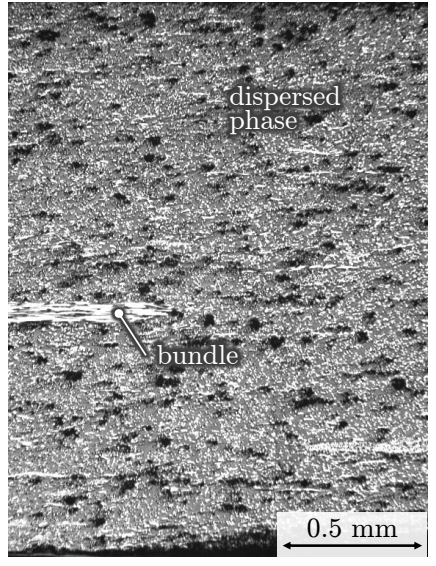

(a) T300-rRCF.

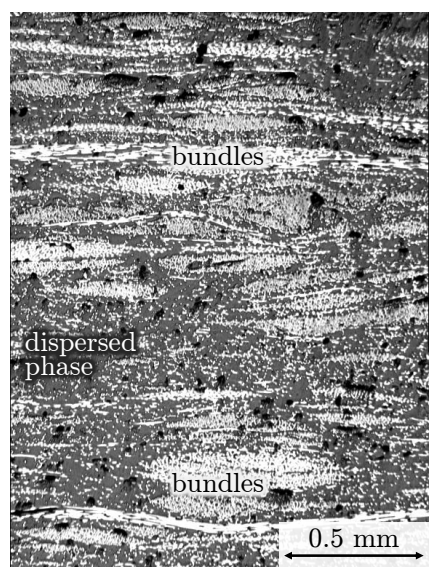

(b) T300-rMIT.

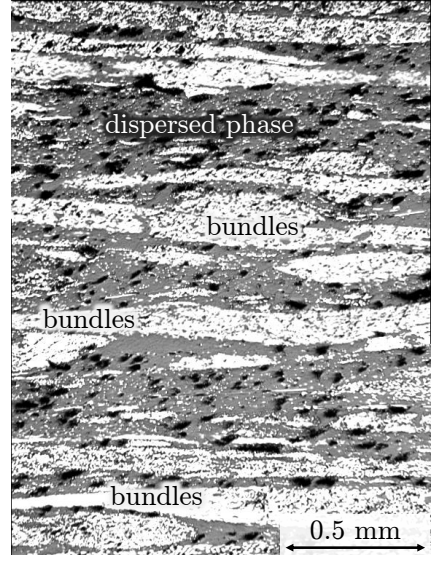

(c) T800-rMIT.

Figure 11: Micrographs of through-the-thickness cross sections of the three recycled composites under analysis.

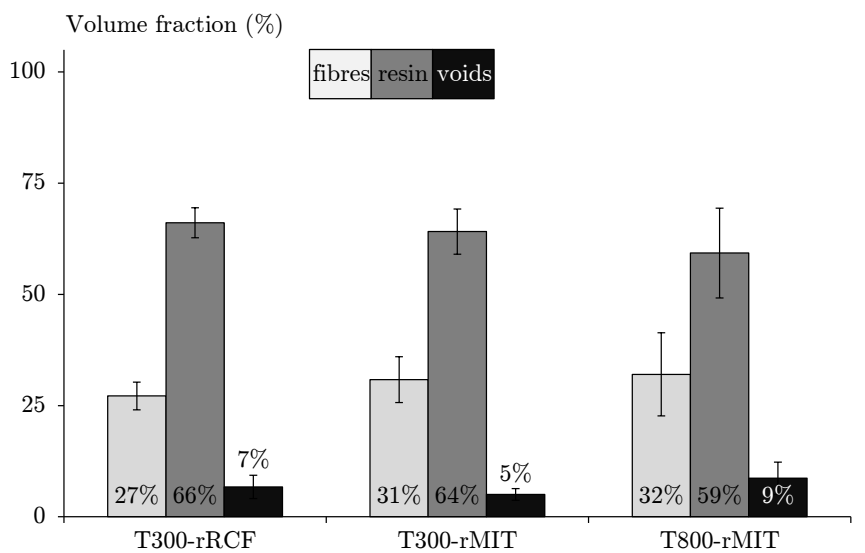

Figure 12: Constituent volume fraction of the recycled composites tested. Error bars represent the standard deviation. 


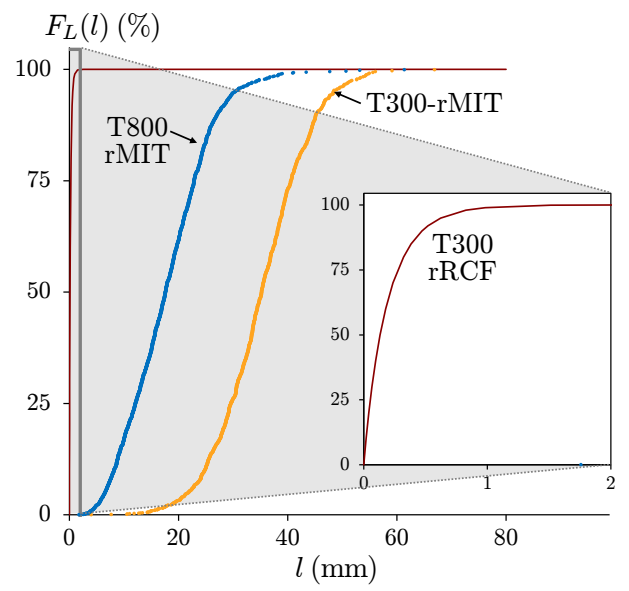

(a) Length distribution.

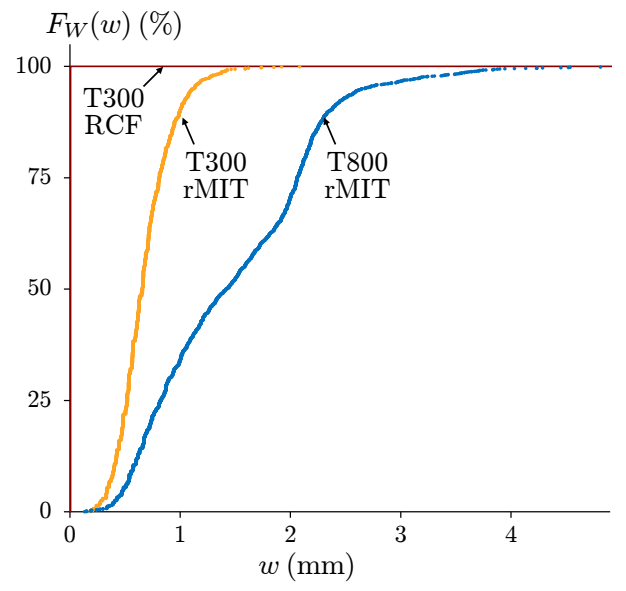

(b) Width distribution.

Figure 13: Characterisation of the architecture of the three rCFRPs. Distributions for the T300-rRCF material were estimated from a similar material and correspond to the dispersed phase only [5].

Table 6: Architecture characterisation.

\begin{tabular}{|c|c|c|c|c|c|c|c|}
\hline \multirow[b]{2}{*}{ Material } & \multicolumn{3}{|c|}{ Length distribution } & \multicolumn{3}{|c|}{ Width distribution } & \multirow{2}{*}{$\begin{array}{c}\text { Aspect } \\
\text { ratio } \\
\quad t / w \\
\end{array}$} \\
\hline & $\begin{array}{l}\left.l_{\mathrm{m}}{ }^{\star}\right) \\
(\mathrm{mm})\end{array}$ & $\begin{array}{c}m_{l} \\
(-)\end{array}$ & $\begin{array}{c}l_{0} \\
(\mathrm{~mm})\end{array}$ & $\begin{array}{l}\bar{w}^{(\dagger)} \\
(\mathrm{mm})\end{array}$ & $\begin{array}{l}m_{w} \\
(-)\end{array}$ & $\begin{array}{c}w_{0} \\
(\mathrm{~mm})\end{array}$ & \\
\hline$\overline{\mathrm{T} 300-\mathrm{rRCF}}{ }^{(\S)}$ & 0.199 & 0.94 & 0.194 & 0.007 & n.a. & n.a. & 1 \\
\hline T300-rMIT & 35.2 & 5.02 & 38.3 & 0.68 & 3.59 & 0.75 & 0.14 \\
\hline T800-rMIT & 17.7 & 2.70 & 19.9 & 1.49 & 2.28 & 1.65 & 0.07 \\
\hline
\end{tabular}

${ }^{(\star)}$ Expected value of Weibull distribution.

(†) Average of raw data.

( ) Estimated from the mass of bundles measured, assuming fibre density $\rho^{f}=1.78 \mathrm{~g} / \mathrm{cm}^{3}$ and content $V^{\mathrm{f}}=60 \%$ in dry bundles.

(§) Data calculated from Wong et al. [5].

The differences between the architectures in the three materials are quantified in Figure 13; only the dispersed phase is considered for T300-rRCF. For each material, bundle lengths and widths are well represented by Weibull distributions; parameters fitted through the least squares method are shown in Table 6. Assuming elliptical bundle cross-sections, it is estimated that bundles in the T300-rMIT are thicker than those in T800-rMIT; no correlation was found between bundle length and width in either material.

\subsubsection{Fracture toughness}

Figure 14 presents the R-curves measured for the three materials; only specimens which exhibited reasonably straight crack propagation are included. No significant difference was found between orthotropic directions (1 and 2) in materials T300-rMIT and T800-rMIT, which supports the assumption of in-plane isotropy. Moreover, all R-curves for material T800-rMIT fit in the same experimental cloud, regardless of the specific specimen geometry used (Fig- 
ure 14); this verifies the requirement of in-plane scale-independence for valid measurements of fracture toughness.

Material T300-rRCF presented the lowest toughness values (even along the preferential fibre direction), followed closely by material T300-rMIT. However, material T800-rMIT is remarkably tougher, especially considering that the process zone was not fully developed and steady-state propagation had not been reached at $\Delta a=60 \mathrm{~mm}$ (meaning that the steadystate fracture toughness of this material actually exceeds $40 \mathrm{~kJ} / \mathrm{m}^{2}$ ).

The R-curves of representative specimens are mapped with fracture surfaces in Figure 14. Going from material T300-rRCF to T300-rMIT and T800-rMIT, it becomes evident that, as the architecture becomes coarser, failure surfaces become more irregular — with broken and pulled-out bundles — and the fracture toughness increases significantly.

\section{Discussion}

\subsection{Analysis of recycling processes and reclaimed fibres}

The quality of recycled fibres was thoroughly assessed, and the results were extremely positive in all domains: all fibre types were reasonably clean and showed no signs of damage (Figures 4 and 5), and retained most tensile strength of their virgin precursors (Figure 6). Figure 9 also suggests a good fibre-matrix interfacial quality; the results obtained for T300rMIT and T800-rMIT are indicative only (as they correspond to respectively 2 and 1 valid experiments), but they do indicate similar interfacial properties for recycled and virgin materials. This corroborates previous studies [22] concluding that re-sizing is not necessary from a micromechanical point of view.

Results show that both ELG-RCF and MIT-RCF's pilot-plant pyrolysis processes are capable of recovering recycled fibres with similar quality to virgin precursors. Nevertheless, reclaiming end-of-life waste is clearly more challenging than recycling uncured manufacturing waste, as the former case yielded fibres with more residue and lower strength retention (Figures 5 and 6). The uneven distribution of residual matrix on the surface of T800-rMIT fibres (also seen in recycled woven composites [22]) suggests that recycling thicker panels or $3 \mathrm{D}$ shapes will likely be problematic.

Finally, it must be noticed that all recyclates analysed in this paper were recovered through pilot-plant operations, but scaling-up towards an industrial process represents a significant challenge [25]. This is perfectly illustrated by the impeccable performance of the T300-rRCF fibres, as opposed to the much more modest results reported in a previous analysis of woven rCFRP with fibres recovered by the same process but implemented at a continuous scale [22].

\subsection{Strength distribution of recycled fibres and size effects}

All recycled fibres exhibited higher strength retention at the $20 \mathrm{~mm}$ gauge length than at $10 \mathrm{~mm}$ (Figure 6); this is unlike what is commonly reported in the literature $[22,24]$, although 


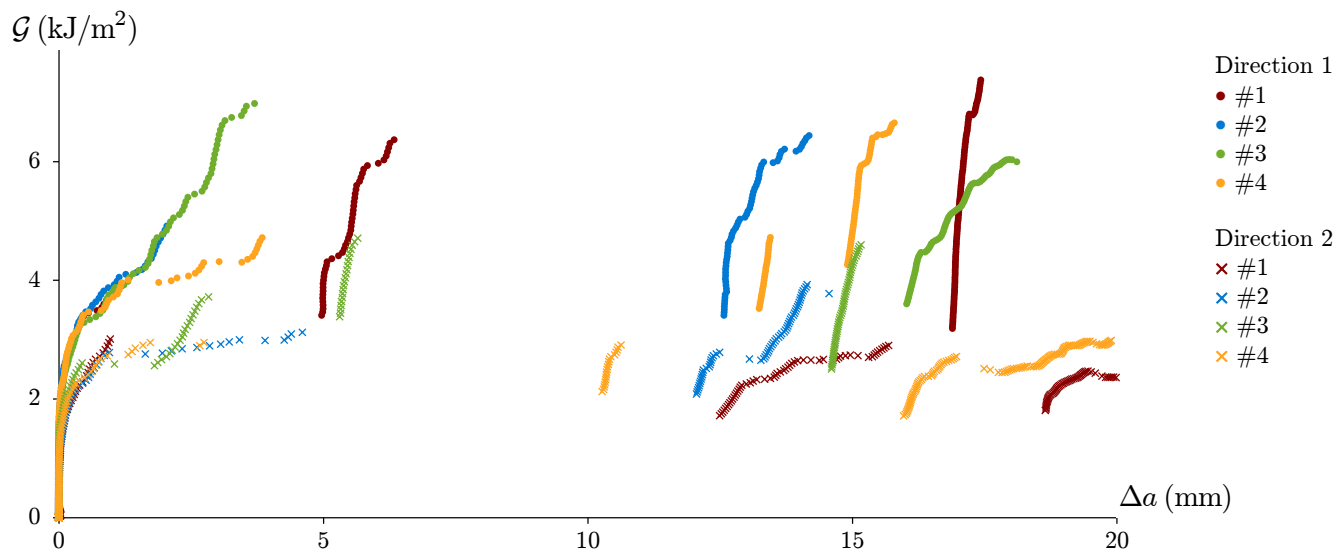

(a) T300-rRCF.

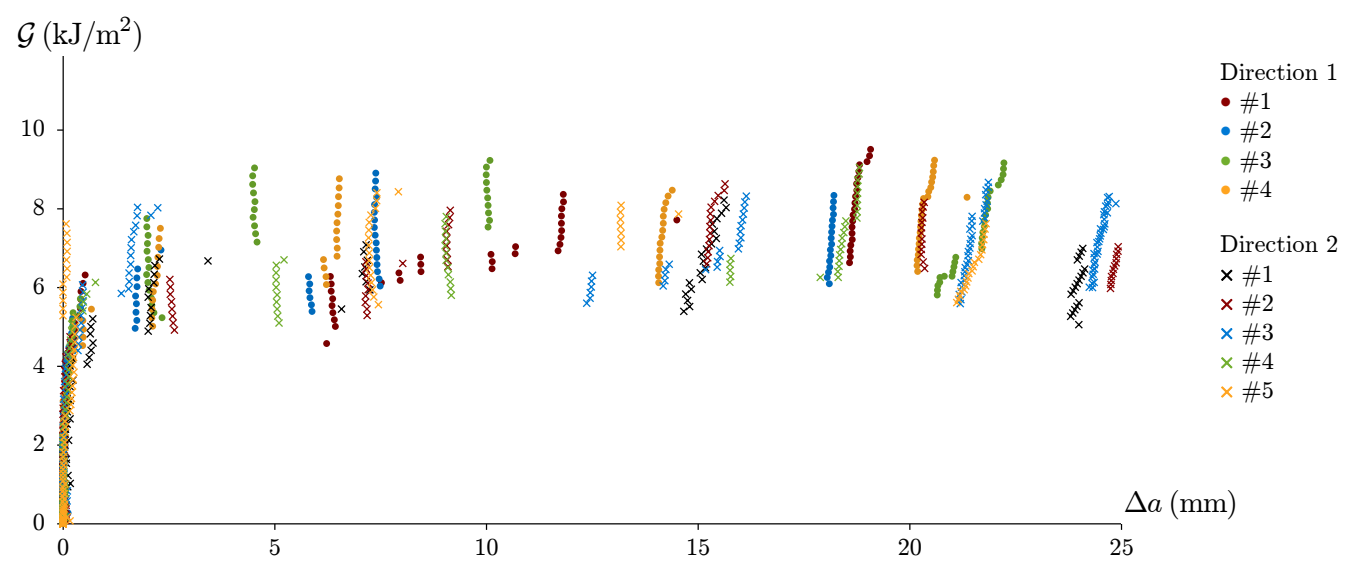

(b) T300-rMIT.

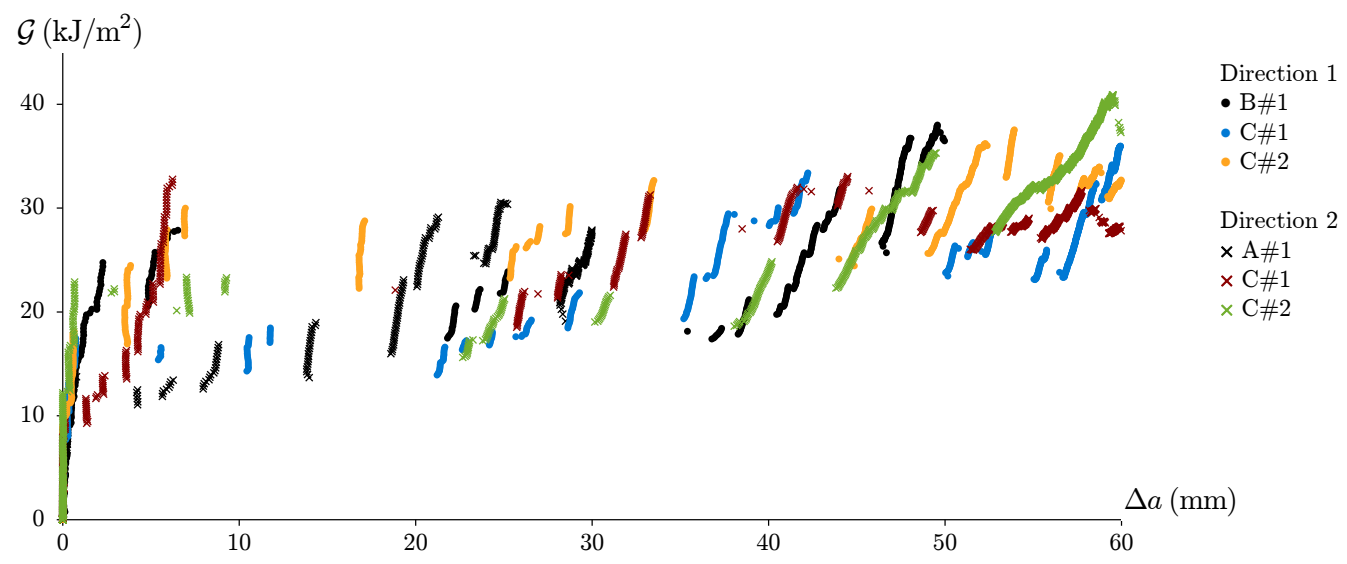

(c) T800-rMIT.

Figure 14: Results from the CT tests. 


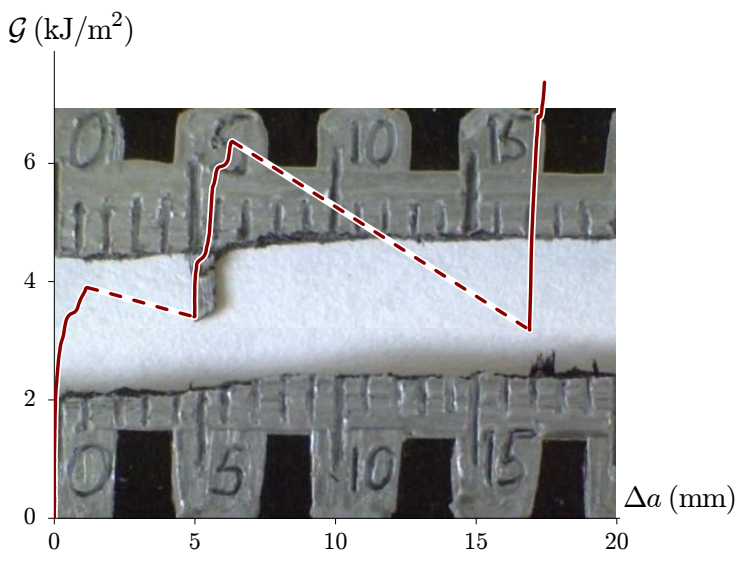

(a) T300-rRCF (specimen 1\#1).

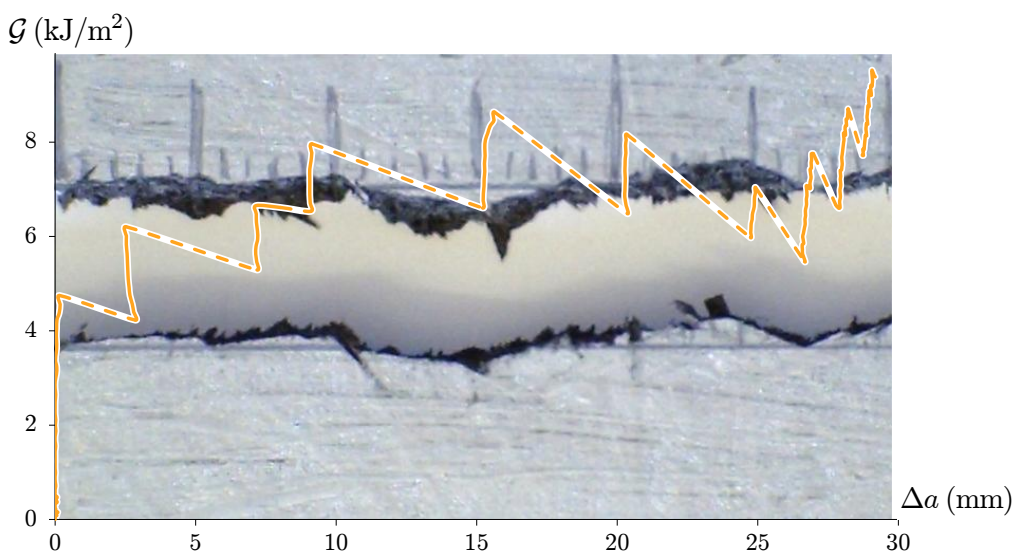

(b) T300-rMIT (specimen 2\#2).

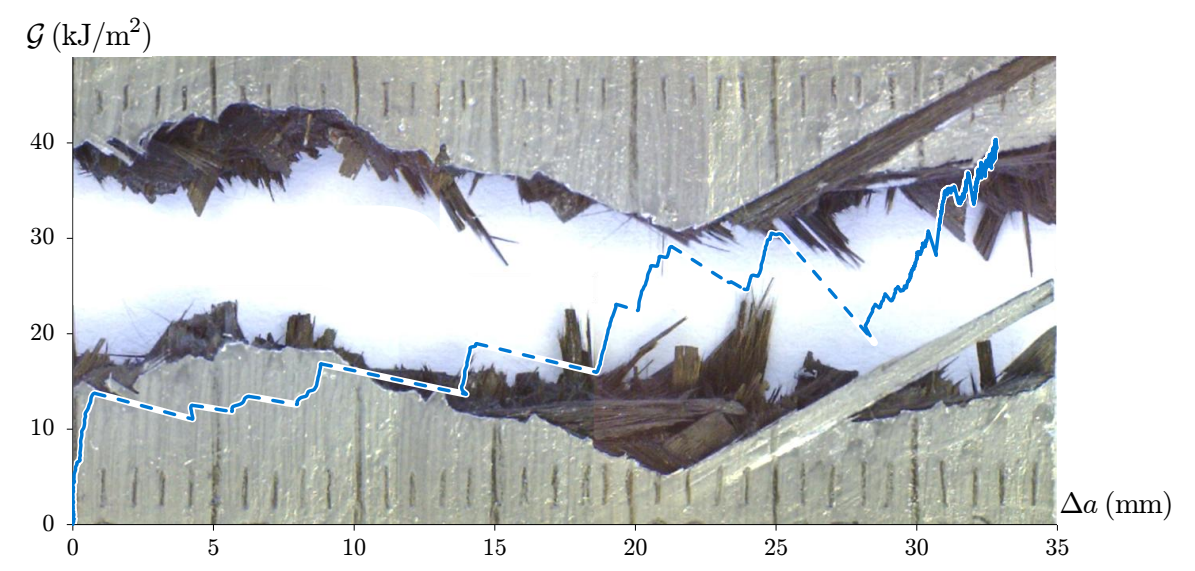

(c) T800-rMIT (specimen A-2\#1).

Figure 15: Representative mapping of fracture surfaces with R-curves obtained through CT tests (all images are at the same scale). 
it has been observed in other recyclates [25]. One possible explanation is that of biased sampling of the recycled fibres towards stronger filaments; all reclaimed fibres went through heavy processing during preforming, and it is possible this fractured the longer filaments at the weakest points (which were thus excluded from the tests).

In addition, the different Weibull moduli obtained directly from fibre strength distributions and from size-effects (Table 2) question the applicability of Weibull-based weakest-link theory to model the strength of individual filaments. Some researchers defend that fibres have nonWeibull strength distributions [43, 44], while others suggest that testing induces spurious effects [33]. Recycled fibres can indeed have more than one population of defects, but they can also be affected differently by the sampling process and testing procedures.

While the fibre strength distributions shown in Table 2 and Figures 7-8 are inconclusive regarding the actual shape of single-fibre strength distributions and the nature of size effects, the averages shown in Figure 6 do provide a solid ground for comparing the virgin and recycled fibres.

\subsection{Effect of architecture on stiffness and strength}

All three rCFRPs present similar stiffness (Figure 1a). However, taking into account that (i) material T300-rRCF is anisotropic, (ii) T800 fibres are stiffer than T300, and (iii) MTM57 resin is stiffer than RTM-6, the architecture of T300-rMIT material (with fine bundles) comes as the most favourable for the overall modulus of the recyclates, due to the large aspect ratio of reinforcing units (Table 6).

Material T300-rMIT exhibited the lowest variability in all properties measured, thus suggesting an advantage of fine bundled architectures in terms of reliability. The large variability in modulus of the T800-rMIT material is likely caused by its heterogeneous and coarse architecture; similar conclusions have been reported for state-of-the-art prepreg-based discontinuous composites $[18,45]$. Nevertheless, the variability measured in the coarsest recycled material (T800-rMIT) is still lower than that of virgin discontinuous carbon-fibre composites (based on chopped prepreg [17] or directed preforms [18], see Figure 1).

Figure 1b shows that large bundles are detrimental to the strength of rCFRPs (although the different matrices used in the filamentised and bundled cases could also have influenced the results). Failure typically initiates at large inhomogeneities due to an energetic size effect [46], even if the aspect ratio is kept constant. Moreover, material T800-rMIT has the highest void content (Figure 12), probably due to the presence of residual matrix (which obstructs impregnation [22]).

Under compression (Figure 1c), materials T300-rMIT and T800-rMIT are consideralby weaker than T300-rRCF. The previous considerations regarding the influence of reinforcement size on tensile strength apply to compression as well; however, in this case, the effect of a different resin (and fibre-matrix interface) is likely stronger. 
Most literature on virgin Short-Fibre Reinforced-Polymers (SFRPs) shows that filamentised materials exhibit superior mechanical properties [15, 16]; this was verified here for strength. However, it has also been reported that filamentised materials require higher moulding processes [16], which is likely to increase fibre failure during manufacturing - thus degrading the aspect ratio of reinforcing units and, consequently, the stiffness of the composites. For this reason, the presence of bundles in recycled composites not only reduces fibre breakage during manufacturing, as it also increases the attainable fibre content at reasonable moulding pressures.

Even though research on virgin discontinuous fibre composites is insightful for the recycled counterparts, results from the two fields are not completely interchangeable due to a complex relation between presence of bundles, residual matrix and fibre strength in the latter. Studies focusing specifically on rCFRPs are thus necessary to improve the understanding of their mechanical response.

\subsection{Effect of architecture on toughening mechanisms}

The characterisation of architectures (Figure 13) and CT results (Figure 14) evidence a clear correlation between the scale of reinforcing units and the fracture toughness or damage tolerance of a composite. The fracture surfaces of the recyclates evidence pulled-out bundles in locally tougher regions (Figure 15a) and overall tougher rCFRPs (Figure 15c), illustrating the importance of the mentioned size effect.

Similar observations on virgin SFRPs have been reported in the literature $[10-15,17,18]$. Size effects on pull-out of reinforcing units have been consistently identified by most authors as the main toughening mechanism in composites with coarse architecture.

Moreover, recent observations of size effects in the toughness of UD composites [47] suggest that thicker bundles are also intrinsically tougher than thinner ones (and than single fibres). This correlates well with the presence of broken bundles in the surfaces shown in Figure 15, and is strongly supported by the fractal defibrillation patterns identified in bundles [9].

Altogether, the fracture toughness of rCFRPs is largely affected by the reinforcement architecture; a coarser microstructure leads to tougher composites, due to size effects on the energy dissipated during both pull-out and bundle failure. While materials T300-rRCF and T300rMIT - with a finer microstructure - are more brittle than most metals $\left(\mathcal{G}<10 \mathrm{~kJ} / \mathrm{m}^{2}\right)$, the bundled T800-rMIT materials (with $\mathcal{G} \approx 40 \mathrm{~kJ} / \mathrm{m}^{2}$ ) is significantly tougher than a structural aluminium alloy (with $\mathcal{G} \approx 20 \mathrm{~kJ} / \mathrm{m}^{2}$ ).

\subsection{Optimisation of recycling routes}

This study highlights a strong relation between the architecture of a rCFRP and its mechanical performance. On the one hand, the presence of fibre bundles weakens recycled composites (Figure 1b); however, recycled materials are unlikely to be used in strength-critical 
structures, thus making this drawback inconsequential for most realistic cases. For stiffness and toughness driven applications, on the other hand, accepting fibre bundles in rCFRPs would present the following advantages:

- Significantly tougher composites. Comparing material T300-rRCF (with a dispersed phase and $\mathcal{G} \approx 3 \mathrm{~kJ} / \mathrm{m}^{2}$ ) with material T800-rMIT (with a coarse architecture and $\mathcal{G}>40 \mathrm{~kJ} / \mathrm{m}^{2}$ ), it is concluded that a coarser reinforcement can boost the fracture toughness of a rCFRP by more than one order of magnitude.

- Composites with higher fibre content. Filamentised preforms have a high loft, thus requiring very high moulding pressures to achieve reasonable reinforcement contents, at the expense of fibre fracture. Consequently, bundled architectures can also feature larger reinforcement aspect ratios, which improves the overall rCFRP stiffness (compare materials T300-rRCF and T300-rMIT in Figure 1a).

- Higher fibre-strength retention. Bundles are a natural consequence of residual resin, which results from soft reclamation cycles that inflict little fibre damage. This is particularly important for industrial processes, which will most likely operate in non-ideal conditions $[22]$;

- Lower recycling costs. Residual resin is favoured by shorter and lower-temperature reclamation cycles, which require less energy. Moreover, manufacturing highly filamentised composites requires additional intermediate steps (e.g. disentanglement stage for material T300-rRCF).

While the benefits of bundled configurations are clear from this study, some challenges require further work. Coarse architectures have inherently larger variability [45], as shown in Figure 1 (the three recycled materials presented nevertheless lower variability than discontinuous virgin composites). It is also necessary to investigate how bundle size can be controlled and the presence of residual matrix minimised; the latter aspect is particularly important to facilitate impregnation and reduce void content in coarser materials, thus improving their mechanical performance even further.

\section{Conclusions}

The mechanical response of three rCFRPs was studied experimentally. The analysis highlighted the relations between recycling routes, micromechanical properties, meso-level architectures and the macroscopic response of the recyclates.

The materials investigated comprised different virgin fibre types (T300 and T800), waste sources (uncured manufacturing scrap and end-of-life components) and reclamation processes (pilot-scale pyrolysis by ELG-RCF [19] and MIT-RCF [20]). The quality of the fibres recycled 
from manufacturing waste was comparable to that of the virgin precursors, and over $80 \%$ of fibre strength was recovered in the end-of-life component case.

All composites analysed were composed of epoxy resin reinforced by discontinuous rCFs, but featured very distinct architectures - from nearly fully dispersed short fibres to long and wide bundles; these differences resulted not only from the fibre-reclamation stage, but also from re-manufacturing. This study shows that, while the reinforcement architecture has limited influence on the overall stiffness and strength of rCFRPs, it dramatically affects the fracture toughness.

Preserving fibre bundles during reclamation and re-manufacturing processes toughened the recyclates by more than one order of magnitude. Fracture toughnesses in excess of $\mathcal{G}=40 \mathrm{~kJ} / \mathrm{m}^{2}$ were measured for the rCFRP with the coarsest architecture; this is significantly above the typical value exhibited by some traditional structural materials (e.g. aluminium), and in the same of magnitude as the toughness of continuous cross-ply CFRP laminates (with $\mathcal{G}=30-115 \mathrm{~kJ} / \mathrm{m}^{2}$ ) [48].

These results open a window of opportunity for rCFRPs in toughness or damage-tolerance driven applications (which include any structure with sharp geometric discontinuities). However, before these materials can be used confidently in (non-safety-critical) structures, it is necessary to further understand and be able to predict the relations between micromechanical properties, reinforcement architecture and the overall toughness of the recyclates. This would be useful not only to guide further optimisation of recycling processes, but also to support engineers aiming to design structures with rCFRPs.

\section{Acknowledgements}

The funding from the Portuguese Foundation for Science and Technology (project nr. SFRH/BD/44051/2008) is gratefully acknowledged. The authors are also thankful to Mr Pete E George and Mr William Carberry (from The Boeing Company), and Prof Steve Pickering and Dr Hoong Wong (from the University of Nottingham), for providing the recycled materials analysed, sharing technical data, and many helpful discussions.

\section{References}

[1] S. Pimenta and S. T. Pinho, "Recycling carbon fibre reinforced polymers for structural applications: Technology review and market outlook," Waste Management, vol. 31, no. 2, pp. 378-392, 2011.

[2] S. J. Pickering, "Recycling technologies for thermoset composite materials - current status," Composites Part A, vol. 37, no. 8, pp. 1206-1215, 2006. 
[3] M. A. Janney, W. L. Newell, E. Geiger, N. Baitcher, and T. Gunder, "Manufacturing complex geometry composites with recycled carbon fiber," in SAMPE'09 Conference, (Baltimore, MD, USA), SAMPE, 18-21 May 2009.

[4] M. Szpieg, M. Wysocki, and L. E. Asp, "Reuse of polymer materials and carbon fibres in novel engineering composite materials," Plastics Rubber and Composites, vol. 38, no. 910, pp. 419-425, 2009.

[5] K. H. Wong, S. J. Pickering, T. A. Turner, and N. A. Warrior, "Compression moulding of a recycled carbon fibre reinforced epoxy composite," in SAMPE'09 Conference, (Baltimore, MD, USA), SAMPE, 18-21 May 2009.

[6] T. A. Turner, N. A. Warrior, and S. J. Pickering, "Development of high value moulding compounds from recycled carbon fibres," Plastics, Rubber and Composites, vol. 39, no. 35, pp. 151-156, 2010.

[7] J. P. Heil, J. B. Gavin, P. E. George, and J. J. Cuomo, "Composite panels made from the wetlay process using recycled carbon fiber," in SAMPE 2011 Conference, (Long Beach, CA, USA), SAMPE, 23-26 May 2011.

[8] J. Meredith, S. Cozien-Cazuc, E. Collings, S. Carter, S. Alsop, J. Lever, S. R. Coles, B. M. Wood, and K. Kirwan, "Recycled carbon fibre for high performance energy absorption," Composites Science and Technology, vol. 72, no. 6, pp. 688-695, 2012.

[9] S. Pimenta, S. T. Pinho, P. Robinson, K. Wong, and S. J. Pickering, "Mechanical analysis and toughening mechanisms of a multiphase recycled CFRP," Composites Science and Technology, vol. 70, no. 12, pp. 1713-1725, 2010.

[10] M. Fila, C. Bredin, and M. R. Piggott, "Work of fracture of fiber-reinforced polymers," Journal of Materials Science, vol. 7, no. 9, pp. 983-988, 1972.

[11] J.-K. Kim and Y.-W. Mai, "High strength, high fracture toughness fibre composites with interface control - A review," Composites Science and Technology, vol. 41, no. 4, pp. 333-378, 1991.

[12] J. K. Kim and Y. W. Mai, "Fracture of CFRP containing impregnated fiber-bundles," Composites Science and Technology, vol. 49, no. 1, pp. 51-60, 1993.

[13] M. R. Piggot, "Mesostructures and their mechanics in fibre composites," Advanced Composite Materials, vol. 6, no. 1, pp. 75-81, 1996.

[14] J. Lindhagen and L. A. Berglund, "Notch sensitivity and damage mechanisms of glass mat reinforced polypropylene," Polymer Composites, vol. 18, no. 1, pp. 40-47, 1997.

[15] D. R. Mulligan, S. L. Ogin, P. A. Smith, G. M. Wells, and C. M. Worrall, "Fibrebundling in a short-fibre composite: 1 . review of literature and development of a method for controlling the degree of bundling," Composites Science and Technology, vol. 63, no. 5, pp. $715-725,2003$. 
[16] L. T. Harper, T. A. Turner, N. A. Warrior, and C. D. Rudd, "Characterisation of random carbon fibre composites from a directed fibre preforming process: The effect of tow filamentisation," Composites Part A, vol. 38, no. 3, pp. 755-770, 2007.

[17] P. Feraboli, E. Peitso, T. Cleveland, P. Stickler, and J. Halpin, "Notched behavior of prepreg-based discontinuous carbon fiber/epoxy systems," Composites Part A, vol. 40, no. 3, pp. 289-299, 2009.

[18] C. Qian, L. T. Harper, T. A. Turner, and N. A. Warrior, "Notched behaviour of discontinuous carbon fibre composites: Comparison with quasi-isotropic non-crimp fabric," Composites Part A, vol. 42, no. 3, pp. 293-302, 2011.

[19] ELG Carbon Fibre Ltd. www.elgcf.com, last accessed March 2013.

[20] Materials Innovation Technologies - Reengineered Carbon Fiber, LCC. http://mitrcf . com, last accessed March 2013.

[21] L. O. Meyer, K. Schulte, and E. Grove-Nielsen, "CFRP-recycling following a pyrolysis route: Process optimization and potentials," Journal of Composite Materials, vol. 43, no. 9, pp. 1121-1132, 2009.

[22] S. Pimenta and S. T. Pinho, "The effect of recycling on the mechanical response of carbon fibres and their composites," Composite Structures, vol. 94, no. 12, pp. 3669-3684, 2012.

[23] J. Howarth and M. Jeschke, "Advanced non-woven materials from recycled carbon fibre," in Carbon Fibre Recycling and Reuse Conference, (Hamburg, Germany), IntertechPira, 3-4 November 2009.

[24] J. P. Heil, M. J. Hall, D. R. Litzenberger, R. Clearfield, J. J. Cuomo, P. E. George, and W. L. Carberry, "A comparison of chemical, morphological and mechanical properties of various recycled carbon fibers," in SAMPE'09 Conference, (Baltimore, MD, USA), SAMPE, 18-21 May 2009.

[25] J. P. Heil, D. R. Litzenberger, and J. J. Cuomo, "A comparison of chemical, morphological, and mechanical properties of carbon fibers recovered from commercial recycling facilities," in SAMPE 2010 Conference, (Seattle, WA, USA), SAMPE, 17-20 May 2010.

[26] S. Panesar, "Converting composite waste into high quality reusable carbon fibre," in JEC Composites Show, (Paris, France), JEC Composites, 24-26 March 2009.

[27] Toray Carbon Fibers America, Inc, "Torayca T300 Data Sheet - Technical Data Sheet No. CFA-001." http://www.toraycfa.com/pdfs/T300DataSheet.pdf, last accessed December 2012.

[28] Umeco Structural Materials (Derby) Ltd., "Umeco MTM 57 Series Prepreg System - ODS1075/03.12/8a." http://www. umeco.com/ /media/Products/Datasheets/ Structural/Epoxy\%20prepregs/MTM57_series\%20pdf.pdf, last accessed December 2012. 
[29] Hexcel Corporation, "HexFlow RTM 6 Product Data - Publication ITA 065e." http: //www.hexcel.com/Resources/DataSheets/RTM-Data-Sheets/RTM6_global.pdf, last accessed December 2012.

[30] Toray Carbon Fibers America, Inc, "Torayca T800H Data Sheet - Technical Data Sheet No. CFA-007." http://www.toraycfa.com/pdfs/T800HDataSheet.pdf, last accessed December 2012.

[31] P. E. George, "Boeing Research and Technology." Personal communication, 2011.

[32] British Standards Institution, "Implementation of ISO 11566:1996, Carbon fibre - Determination of the tensile properties of single-filament specimens," 1996.

[33] E. G. Stoner, D. D. Edie, and S. D. Durham, "An end-effect model for the single-filament tensile test," Journal of Materials Science, vol. 29, no. 24, pp. 6561-6574, 1994.

[34] A. Bismarck, A. Menner, M. E. Kumru, A. S. Sarac, M. Bistritz, and E. Schulz, "Poly(carbazole-co-acrylamide) electrocoated carbon fibers and their adhesion behavior to an epoxy resin matrix," Journal of Materials Science, vol. 37, no. 3, pp. 461-471, 2002 .

[35] P. Chua and M. Piggott, "The glass fibre-polymer interface: II - Work of fracture and shear stresses," Composites Science and Technology, vol. 22, no. 2, pp. 107-119, 1985.

[36] W. Gilchrist, Statistical Modelling with Quantile Functions. Chapman and Hall/CRC, USA, 2000.

[37] S.-Y. Fu, Y.-W. Mai, E. C.-Y. Ching, and R. K. Y. Li, "Correction of the measurement of fiber length of short fiber reinforced thermoplastics," Composites Part A, vol. 33, no. 11, pp. 1549-1555, 2002.

[38] S. Pinho, P. Robinson, and L. Iannucci, "Fracture toughness of the tensile and compressive fibre failure modes in laminated composites," Composites Science and Technology, vol. 66, no. 13, pp. 2069-2079, 2006.

[39] M. J. Laffan, S. T. Pinho, P. Robinson, and L. Iannucci, "Measurement of the in situ ply fracture toughness associated with mode I fibre tensile failure in FRP. Part I: Data reduction," Composites Science and Technology, vol. 70, no. 4, pp. 606-613, 2010.

[40] M. J. Laffan, S. T. Pinho, and P. Robinson, "Mixed-mode translaminar fracture of CFRP: Failure analysis and fractography," Composite Structures, vol. 95, no. 1, pp. 135$141,2013$.

[41] L.-M. Zhou, J.-K. Kim, and Y.-W. Mai, "On the single fiber pull-out problem: effect of loading method," Composites Science and Technology, vol. 45, no. 2, pp. 153-160, 1992.

[42] X. Zhang, H.-Y. Liu, Y.-W. Mai, and X.-X. Diao, "On steady-state fibre pull-out - I — The stress field," Composites Science and Technology, vol. 59, no. 2, pp. 2179-2189, 1999. 
[43] C. P. Beetz, "The analysis of carbon-fiber strength distributions exhibiting multiplemodes of failure," Fibre Science and Technology, vol. 16, no. 1, pp. 45-59, 1982.

[44] I. J. Beyerlein and S. L. Phoenix, "Statistics for the strength and size effects of microcomposites with four carbon fibers in epoxy resin," Composites Science and Technology, vol. 56, no. 1, pp. 75-92, 1996.

[45] P. Feraboli, E. Peitso, T. Cleveland, and P. Stickler, "Modulus measurement of prepregbased discontinuous carbon fiber/epoxy systems," Journal of Composite Materials, vol. 43, no. 19, pp. 1947-1965, 2009.

[46] Z. P. Bažant, "Size effect on structural strength: a review," Archive of Applied Mechanics, vol. 69, no. 9-10, pp. 703-725, 1999.

[47] M. J. Laffan, S. T. Pinho, P. Robinson, and L. Iannucci, "Measurement of the in situ ply fracture toughness associated with mode I fibre tensile failure in FRP. Part II: Size and lay-up effects," Composites Science and Technology, vol. 70, no. 4, pp. 614-621, 2010.

[48] M. J. Laffan, S. T. Pinho, P. Robinson, and A. J. McMillan, "Translaminar fracture toughness testing of composites: A review," Polymer Testing, vol. 31, no. 3, pp. 481-489, 2012 . 